\title{
CONSTRUCCIÓN DE CONTENIDOS PARA LA ENSEÑANZA VIRTUAL: RETOS COYUNTURALES EN EL CONFINAMIENTO
}

Norman-Acevedo, Eduardo; Daza-Orozco, Carlos Eduardo

CONSTRUCCIÓN DE CONTENIDOS PARA LA ENSEÑANZA VIRTUAL: RETOS COYUNTURALES EN EL CONFINAMIENTO

PANORAMA, vol. 14, núm. 27, 2020

Politécnico Grancolombiano, Colombia

Disponible en: http://www.redalyc.org/articulo.oa?id=343964051001

DOI: https://doi.org/10.15765/pnrm.v14i27.1517

https://journal.poligran.edu.co/index.php/panorama/about/submissions\#copyrightNotice

Esta obra está bajo una Licencia Creative Commons Atribución-NoComercial-SinDerivar 4.0 Internacional. 


\title{
Editorial
}

\section{CONSTRUCCIÓN DE CONTENIDOS PARA LA ENSEÑANZA VIRTUAL: RETOS COYUNTURALES EN EL CONFINAMIENTO}

\author{
CONSTRUCTION OF CONTENT FOR VIRTUAL \\ EDUCATION: TEMPORARY CHALLENGES IN \\ CONFINEMENT \\ CONSTRUÇÃO DE CONTEÚDOS PARA A \\ EDUCAÇÃO VIRTUAL: DESAFIOS TEMPORÁRIOS \\ NO CONFINAMENTO
}

PANORAMA, vol. 14, núm. 27, 2020

Politécnico Grancolombiano, Colombia

Recepción: 01 Junio 2020

Aprobación: 15 Junio 2020

DOI: https://doi.org/10.15765/ pnrm.v14i27.1517

Redalyc: http://www.redalyc.org/ articulo.oa?id $=343964051001$

https://journal.poligran.edu.co/ index.php/panorama/about/ submissions\# copyrightNotice CC BY-NC-ND
Eduardo Norman-Acevedo ednorman@poligran.edu.co Institución Universitaria Politécnico Grancolombiano., Colombia

Carlos Eduardo Daza-Orozco cdaza@poligran.edu.co Institución Universitaria Politécnico Grancolombiano., Colombia

Resumen: Tras la situación de salubridad pública mundial a causa del COVID-19, la educación ha asumido cambios epistemológicos, éticos, sociológicos, pedagógicos, evaluativos e incluso cotidianos, que proponen centrar la reflexión en la educación virtual per se y no desde modelos replicantes de la presencialidad en entornos digitales. El presente artículo versa acerca de la experiencia del Politécnico Grancolombiano y algunos elementos de referencia para la construcción de contenidos para la educación en situación de confinamiento.

Palabras clave: Educación, educación superior, construcción de contenidos, escenarios de aprendizaje.

Abstract: After the situation of global public health due to the COVID-19; Education has assumed epistemological, ethical, sociological, pedagogical, evaluative and even daily changes, which propose to focus the reflection on virtual education per se and not from replicating models of presence in digital environments. The present article deals with the experience of the Politécnico Grancolombiano and some reference elements for the construction of contents for education in a situation of confinement.

Keywords: Education, higher education, content construction, learning scenarios.

Resumo: Após a situação de saúde pública global devido à COVID-19; a educação assumiu mudanças epistemológicas, éticas, sociológicas, pedagógicas, avaliativas e mesmo diárias, que se propõem concentrar a reflexão na educação virtual per se e não a partir da replicação de modelos de presença em ambientes digitais. O presente artigo aborda a experiência do Politécnico Grancolombiano e alguns elementos de referência para a construção de conteúdos para a educação numa situação de confinamento.

Palavras-chave: Educação, ensino superior, construção de conteúdos, cenários de aprendizagem. 


\section{INTRODUCCIÓN}

El Politécnico Grancolombiano, como Institución Universitaria con 40 años al servicio de la educación colombiana, inició su modelo de educación virtual en el año 2008, desarrollando una plataforma robusta que contempla un modelo educativo innovador y la generación de contenidos pedagógicos únicos para la enseñanza virtual de una comunidad que en la actualidad acoge a más de 45.000 estudiantes en la virtualidad y 9.612 pertenecientes a la modalidad presencial, distribuidos en 101 programas académicos activos, de los cuales 67 pertenecen al modelo virtual y los 34 restantes, al presencial. El reto del Poli, como Institución de Educación Superior, siempre se enmarcó en la democratización del acceso y cualificación de profesionales en diversas áreas del conocimiento para el desarrollo regional (Institución Universitaria Politécnico Grancolombiano, 2020).

A dicho reto se sumó, de manera jamás imaginada, la situación mundial de salubridad pública a causa del SARS-CoV2, el cual conglomeró a los habitantes del planeta a confinarse en sus hogares, reduciendo cualquier posibilidad de contacto humano y haciendo que la educación sufriese una conmoción en aspectos epistemológicos, éticos, sociológicos, pedagógicos, evaluativos e incluso cotidianos, que dieron como eje de reflexión pensar la virtualidad per se y no desde la presencialidad como leitmotiv tecnologicista del aula tradicional. Ajustes en estructuras curriculares, dispositivos para la comunicación, y reordenamiento de tiempos de aprendizaje se dieron en la imperiosa inmediatez del avance de la pandemia, promoviendo un nuevo ajuste a modelos pedagógicos en donde los aspectos nucleares de acción educativa están basados en la "escuela-hogar" y se alejan de los paradigmas tradicionales de la "escuelainstitución social”.

Los primeros días de puesta en marcha de la "escuela-hogar" en confinamiento fue marcada por una presunta mimesis de la «teoría del caos» (Bravo-Torres et al., 2015): "estadios caóticos" fueron relacionados con el acceso tecnológico, tanto en dispositivos como en oportunidades de conexión a internet de estudiantes y docentes, originando a posteriori el resurgimiento de alternativas tales como televisión y radio educativa. Por otra parte, "estadios inestables" (Janura, Bizovska, Svoboda, Cerny, \& Zemkova, 2017), fueron evidentes en la alfabetización digital de las diversas comunidades educativas, donde las prácticas docentes libres de tecnología fueron sustituidas rápidamente en la implementación de infraestructuras basadas en el uso de video-llamadas, LMS, salas colaborativas, pizarras virtuales, entre otras, que dieron paso -por una parte- a la explosión de cursos de actualización en herramientas digitales, tutoriales audiovisuales y a críticas tradicionalistas de la enseñanzaaprendizaje mediada por tecnología: ¿Realmente se está aprendiendo en la virtualidad? ¿La calidad de la educación actual es adecuada?

Bajo un "estadio estable" nos encontrábamos algunas instituciones que, desde la constancia, la dedicación y el ejercicio sistemático de prácticas educativas en virtualidad tuvimos que realizar algunos pequeños 
ajustes en cobertura, ya que desde la experiencia adquirida se contaba con los entornos virtuales adecuados y los contenidos actualizados. En este panorama, contamos con la fortuna de ser convocados por el "Plan padrino IES", del Ministerio de Educación Nacional, para acompañar procesos de educación presencial asistida por tecnologías de la información a otras instituciones pares que se encontraban inmersas en estadios caóticos o inestables, que al no ser parte de su naturaleza o modalidad no contemplaban una implementación de la virtualidad en el desarrollo de sus procesos académicos.

Como cientistas sociales dedicados a la reflexión educativa pudimos constatar -en el avance de la situación de confinamiento- la conformación de metodologías para el apoyo formativo virtual supeditado a la construcción de ecosistemas de aprendizaje o "escenarios"; el apoyo en sistemas de gestión de aprendizaje (LMS - Learning Management System, acrónimo en inglés); y un cambio en la concepción que se tiene sobre la labor cotidiana del docente. Aquí se incluyen las ideas de equipo formativo .directivos-docentes-estudiantes), en el planteamiento de retos para la consolidación de conocimientos colectivos. (A modo de profundización de estas ideas, se sugiere la revisión de autores como Dewanti, 2016; Geertshuis \& Liu, 2016; Meza-Bolaños, Compañ, \& Satorre, 2019; van de Heyde \& Siebrits, 2019; Veiga, Campos, Braga, \& David, 2016; Cortés et al., 2019; Daza-Orozco, 2015b, 2015a, 2019).

\section{COYUNTURA Y QUEHACER DOCENTE}

Dada la situación presentada, para revisar el desarrollo de la virtualización obligatoria que la coyuntura nos arrojó a afrontar no podemos convertir el quehacer académico en un factor que añade carga emocional a nuestros hogares. Se hace necesario revisar los volúmenes de carga académica y la forma en que estamos haciendo las cosas, para poder establecer un balance entre los tiempos de dedicación y el volumen de actividades propuestas para ser desarrolladas de una manera coherente entre las diferentes asignaturas.

Como es comprensible, la dinámica de requerir trabajo virtual a los estudiantes por parte de los docentes debe estar abrumándolos, de modo que se ha olvidado que la planeación establecida para el desarrollo de las clases presenciales estaba programada para el desarrollo de las clases durante el horario en el aula. Si solicitamos a cada docente el uso de su innovación y pedagogía, sin comunicarse con sus pares, el resultado es el bombardeo de actividades que planean la apropiación del objetivo propuesto en la asignatura individual. Pero claramente el volumen de trabajo solicitado desborda el tiempo de trabajo en el aula al que todos estábamos acostumbrados en la presencialidad; este contexto nos hace pensar en varias situaciones que deben estar dentro de nuestras discusiones.

La construcción de contenido orientado a la virtualidad no es algo que se pueda hacer en corto tiempo, dado que requiere de una planeación, construcción y desarrollo, que no ha sido previsto dada la 
coyuntura. Algunas corrientes de pensamiento recientes se preguntan si los programas de formación de profesores en período de formación deberían hacer más hincapié en preparar mejor a los estudiantes para el aprendizaje en línea, y cuáles serán las consecuencias a largo plazo en la enseñanza de la educación, debido a la actual situación de pandemia (Varea \& González-Calvo, 2020).

El enfoque pedagógico acoge tanto el cognitivismo como el constructivismo. La perspectiva cognitivista (Piaget, 1962, 1968, 1973, 1986), se asume desde la mirada de Piaget, comprendido mediante la acomodación de una estructura de conocimiento para generar nueva información apropiada. El constructivismo recoge, de una parte, el concepto de Piaget referente a la interacción con los objetos de conocimiento; por otra parte, Vygotsky se plantea de la interacción con los otros; y distintos autores indican la importancia de la interacción significativa (Jumaat et al., 2019; Riestenberg, 2020; Shin, Kim, \& Song, 2020). Así que el tutor debe hacer una relación entre lo pedagógico, lo comunicativo y la mediación en línea, a fin de construir un balance (Vygotsky, 2013).

\section{ESCENARIOS DE APRENDIZAJE Y RECURSOS}

El diseño de actividades para el aprendizaje en Educación Virtual requiere de una selección cuidadosa de estructura y rutas que conduzcan al estudiante a la gestión de la información, la comunicación, la interacción en pro de la gestión del conocimiento. También se hace indispensable la participación permanente y activa de la comunicación sincrónica y asincrónica, para lo cual los formadores deben contar con el manejo y apropiación de competencias tutoriales, didácticas y tecnológicas, incluidas las comunicativas e interpersonales en cada una de las anteriores categorías (Institución Universitaria Politécnico Grancolombiano, 2017, p.5).

Hemos comprendido que los escenarios digitales requieren de elementos multimedia y, por lo mismo, es necesario hablar múltiples 'lenguajes'. En este largo camino de la educación virtual no solo los estudiantes han aprendido. Nosotros, en el Politécnico Grancolombiano también hemos adquirido importantes experiencias que nos han ayudado a consolidar este sueño de brindar acceso a educación de calidad para los colombianos. (Norman-Acevedo, 2018)

La virtualización de los contenidos nos plantea, entonces, un nuevo paradigma de la educación asistida por TIC; tiene por objeto facilitar la realización de actividades en cualquier contexto académico, con ayuda de dispositivos de información y teniendo en cuenta las relaciones sociales entre los estudiantes y el tutor para la generación de conocimiento. Esta visión requiere medios para apoyar las experiencias compartidas, aprovechando las capacidades de comunicación e informática de los dispositivos conectados, apoyándose en las comunicaciones directas o por el aprovechamiento de herramientas tecnológicas disponibles (Bravo- 
Torres et al., 2015). Esto plantea al educador un reto y un cambio en su forma de hacer las cosas.

De esta manera, nuestra responsabilidad como formadores, además de la apropiación de los contenidos que estaban previstos para nuestro ejercicio presencial, debe orientarse a un nuevo esfuerzo contingente y metodológico compartido con los estudiantes para potenciar los procesos didácticos y de apropiación.

Quizás a futuro esta experiencia nos resignifique como sociedad la forma de hacer las cosas en el aula, con la posibilidad de apropiar nuevos recursos y experiencias, lo que nos puede convertir en tutores $2.0 \mathrm{y}$ estudiantes 2.0, con nuevas habilidades y destrezas dentro de un entorno blended, potenciado.

\section{REFERENCIAS}

Bravo-Torres, J., López-Nores, M., Blanco-Fernández, Y., Pazos-Arias, J. J., Ramos-Cabrer, M., \& Gil-Solla, A. (2015). An improved virtualization layer to support distribution of multimedia contents in pervasive social applications. Journal of Network and Computer Applications, 51, 1-17. ht tps://doi.org/10.1016/j.jnca.2014.12.003

Cortés, M., Norman-Acevedo, E., \& Rodríguez, D. (2019). Characterization of formative research projects in virtual programs by the Politécnico Grancolombiano. Revista Electronica de Investigacion Educativa, 21(1), 111. https://doi.org/10.24320/REDIE.2019.21.E19.1992

Daza-Orozco, CE. (2015a). Investigación y emprendimiento: experiencias de las Instituciones de Educación Superior Mesa IEST. https://doi.org/10.13140 /RG.2.1.4728.3283

Daza-Orozco, CE. (2015b). La Investigación como proyecto de vida: un acercamiento al quehacer de los Semilleros de Investigación. https://doi.org /10.13140/RG.2.1.2631.1761

Daza-Orozco, CE. (2019). Iniciación científica: conceptualización, metodologías y buenas prácticas (1st ed.; C. E. Daza-Orozco, Ed.). https://doi.org/10.1 3140/RG.2.2.20812.23684

Dewanti, P. (2016). Linking national standards of distance education with e-Learning ecosystem. Journal of Theoretical and Applied Information Technology, 86(3), 382-393. Recuperado de: https://www.scopus.com/inward/record.uri?eid=2-s2.0-849775870 86\&partnerID $=40 \& \mathrm{md} 5=40 \mathrm{ba} 081288 \mathrm{bf3} 32 \mathrm{cc} 7 \mathrm{~d} 3 \mathrm{ebac} 461 \mathrm{~b} 687 \mathrm{a}$

Geertshuis, S., \& Liu, Q. (2016). E-learning ecosystem awareness and professional identity in e-learning technology adoption. In N. J. \& J. A. (Eds.), Proceedings of the European Conference on eLearning, ECEL (Vol. 2016-Janua, pp. 230-238). Recuperado de: https://www.scopus.com/inward/record.uri?eid=2-s2.0-850007978 60\&partnerID $=40 \& \mathrm{md} 5=609 \mathrm{~b} 6434 \mathrm{f} 08 \mathrm{~b} 118 \mathrm{a} 18 \mathrm{e} 480 \mathrm{~d} 87 \mathrm{~d} 633211$

Institución Universitaria Politécnico Grancolombiano. (2017). Tecnologías de la Información y Comunicación, TIC\#: factores, medios y mediaciones.

Institución Universitaria Politécnico Grancolombiano. (2020). Boletín Estádistico 2020. Recuperado de: https://boletinestadistico.poligran.edu .co/programas-academicos.html 
Janura, M., Bizovska, L., Svoboda, Z., Cerny, M., \& Zemkova, E. (2017). Assessment of postural stability in stable and unstable conditions. Acta of Bioengineering and Biomechanics, 19(4), 89-94. https://doi.org/10.5277 /ABB-00832-2017-02

Jumaat, N., Ahmad, N., Abu Samah, N., Ashari, Z., Ali, D., \& Abdullah, A. (2019). Facebook as a platform of social interactions for meaningful learning. International Journal of Emerging Technologies in Learning, 14(4), 151-159. https://doi.org/10.3991/ijet.v14.i04.9363

Meza-Bolaños, D., Compañ, P., \& Satorre, R. (2019). Analysis of Relevant Factors to Measure the Impact of Investment in e-learning Ecosystems in Public Universities (P. M., F. C., \& R. A., Eds.). International Conference on Information Technology and Systems, ICITS 2019, Vol. 918, pp. 781790. https://doi.org/10.1007/978-3-030-11890-7_73

Norman-Acevedo, E. (2018). Rompiendo barreras, 10 años de la Educación virtual en el Politécnico Grancolombiano. (primera; E. Norman-Acevedo, Ed.). Bogotá: Institución Universitaria Politécnico Grancolombiano.

Piaget, J. (1968). Quantification, conservation, and nativism. Science, 162(3857), 976-979. https://doi.org/10.1126/science.162.3857.976

Piaget, J. (1973). The affective unconscious and cognitive unconscious. Journal of the American Psychoanalytic Association, 21(2), 249-261. https://doi.o $\mathrm{rg} / 10.1177 / 000306517302100201$

Piaget, J. (1986). Essay on necessity. Human Development, 29(6), 301-314. ht tps://doi.org/10.1159/000273106

Piaget, J. (1962). Will and action. Bulletin of the Menninger Clinic, 26, 138-145. Recuperado de: https://www.scopus.com/inward/record.uri?eid=2-s2.0-164250523 9\&partnerID $=40 \& \mathrm{md} 5=8398 \mathrm{e} 76205 \mathrm{a} 263787 \mathrm{~d} 7 \mathrm{ef3ddf7726ef7}$

Riestenberg, K. (2020). Meaningful interaction and affordances for language learning at a Zapotec revitalisation programme. Language Learning Journal, 48(3), 316-330. https://doi.org/10.1080/09571736.2020.1722 205

Shin, Y., Kim, D., \& Song, D. (2020). Types and Timing of Scaffolding to Promote Meaningful Peer Interaction and Increase Learning Performance in Computer-Supported Collaborative Learning Environments. Journal of Educational Computing Research, 58(3), 640-661. https://doi.org/10. 1177/0735633119877134

Van de Heyde, V., \& Siebrits, A. (2019). The ecosystem of e-learning model for higher education. South African Journal of Science, 115(5-6). https://doi .org/10.17159/sajs.2019/5808

Varea, V., \& González-Calvo, G. (2020). Touchless classes and absent bodies: teaching physical education in times of Covid-19. Sport, Education and Society, 1-15. https://doi.org/10.1080/13573322.2020.1791814

Veiga, W., Campos, F., Braga, R., \& David, J. (2016). A software ecosystem approach to e-learning domain . In C. C., V. P., W. R.S., \& S. F.A. (Eds.), 12th Brazilian Symposium on Information Systems, SBSI 2016 (pp. 574-584). Recuperado de: https://www.scopus.com/inward/record.uri?eid=2-s2.0-850263496 17\&partnerID $=40 \& \mathrm{md} 5=\mathrm{a} 4643 \mathrm{fa} 9430 \mathrm{fcba} 5 \mathrm{de} 95 \mathrm{~cd} 10 \mathrm{cla} 88 \mathrm{f} 32$

Vygotsky, L. (2013). Experimental research of developing new verbal reflexes according to the mode of linking them to complexes 
(logical memory). Voprosy Psikhologii, (3), 119-125. Recuperado de: https://www.scopus.com/inward/record.uri?eid=2-s2.0-848814435 69\&partnerID $=40 \& \mathrm{md} 5=45 \mathrm{c} 4 \mathrm{a} 5 \mathrm{~b} 0395 \mathrm{abf68b} 191 \mathrm{f} 366 \mathrm{a} 4926 \mathrm{~b} 90$ 